\title{
Model Predictive Control for Spacecraft Rendezvous
}

\author{
Somasundar Kannan ${ }^{1, \star}$, Seyed Amin Sajadi Alamdari ${ }^{1}$, Jan Dentler ${ }^{1}$, \\ Miguel A. Olivares-Mendez ${ }^{1}$, and Holger Voos ${ }^{1}$ \\ ${ }^{1}$ SnT- University of Luxembourg, 6 rue Richard Coudenhove-Kalergi, Kirchberg, L-1359 Luxembourg
}

\begin{abstract}
The current paper addresses the problem of Spacecraft Rendezvous using Model Predictive Control (MPC). The Clohessy-Wiltshire-Hill equations are used to model the spacecraft relative motion. Here the rendezvous problem is discussed by trajectory control using MPC method. Two different scenarios are addressed in trajectory control. The first scenario consist of position control with fuel constraint, secondly the position control is performed in the presence of obstacles. Here the problem of fuel consumption and obstacle avoidance is addressed directly in the cost function. The proposed methods are successfully analysed through simulations.
\end{abstract}

\section{Introduction}

Autonomous operation of Spacecrafts have been a challenging and important problem for many of the space missions undertaken in the recent years. Specially the orbital task requirements with rendezvous and docking are critical in mission involving Autonomous Transfer Vehicle for cargo, On-Orbit Refuelling, Capture of Orbital Debris, Collision Avoidance with Orbital Debris etc. In the future space missions there is an increasing demand for autonomous trajectory control and requires efficient control methods to handle various mission constraints.

In [1] an extensive survey about control of space rendezvous missions can be obtained where as in [2] the author has provided a tutorial on application of Model Predictive Control for Rendezvous mission. In [3], [4] and [5] Model Predictive Control (MPC) was used for rendezvous and docking along with collision avoidance problem using hard constraint method.

With respect to the above discussed papers we have implemented the Model Predictive Control for Spacecraft Rendezvous and Collision avoidance using soft constraint method. In the soft constraint method the collision avoidance constraints are expressed implicitly in the Control Performance cost function. The remainder of the article is organised as follows: First the dynamic model of the Spacecraft Relative motion problem is discussed followed by the Model Predictive Control technique. Finally simulation studies are performed to analyse the proposition using two scenarios such as trajectory control with fuel efficiency and then collision avoidance problem during trajectory control.

\section{Modelling}

Here we will briefly discuss the Clohessy-Wiltshire-Hill [6] equation which are used for the rendezvous problem. But first we will have to define the Hill's frame which can be seen in Figure-1. Here $\vec{R}_{0}$ and $\vec{R}_{1}$ are distances of the reference satellite and second satellite from the central body or Earth. The relative distance between the satellites is given by $\vec{R}_{01}=\vec{R}_{1}-\vec{R}_{0}$. For small values of $\left|\vec{R}_{01}\right|$, the relative acceleration in the rotating frame can be given by [6]

$$
\ddot{\vec{R}}_{01}=\frac{\mu}{R_{0}^{3}}\left[-\vec{R}_{01}+3\left(\frac{\vec{R}_{0}}{R_{0}} \cdot \vec{R}_{01}\right) \frac{\vec{R}_{0}}{R_{0}}\right]+\vec{F}+O\left(R^{2}\right)
$$

where $R_{0}=\left|\vec{R}_{0}\right|, \mu$ is a gravitational constant, $\vec{F}$ is the external force vector. Under the assumption of circular orbit, the linearized Clohessey-Wiltshire-Hill (CW) equation can be used to represent the relative motion of a given spacecraft with respect to a virtual center $[6,7]$ as follows

$$
\begin{aligned}
\ddot{x}-2 n_{0} \dot{y}-3 n_{0}^{2} x & =u_{x} \\
\ddot{y}+2 n_{0} \dot{x} & =u_{y} \\
\ddot{z}+n_{0}^{2} \dot{z} & =u_{z}
\end{aligned}
$$

where $[x, y, z]^{T}$ are the relative states for the spacecraft, $n_{0}=\sqrt{\left(\mu / R_{0}^{3}\right)}$ is the angular velocity of the reference orbit, $R_{0}$ as described before is the radius of the reference orbit, $\left[u_{x}, u_{y}, u_{z}\right]^{T}$ are the external forces.

\section{Model Predictive Control}

In the current section we will briefly discuss the Model Predictive Control (MPC) method. In the MPC implementation an internal model is used to predict the behaviour of the system in the horizon $\tau=[t, t+T]$ at each time instant $t$. The control input is obtained by minimization of the

\footnotetext{
^e-mail: somasundar.kannan@uni.lu
} 


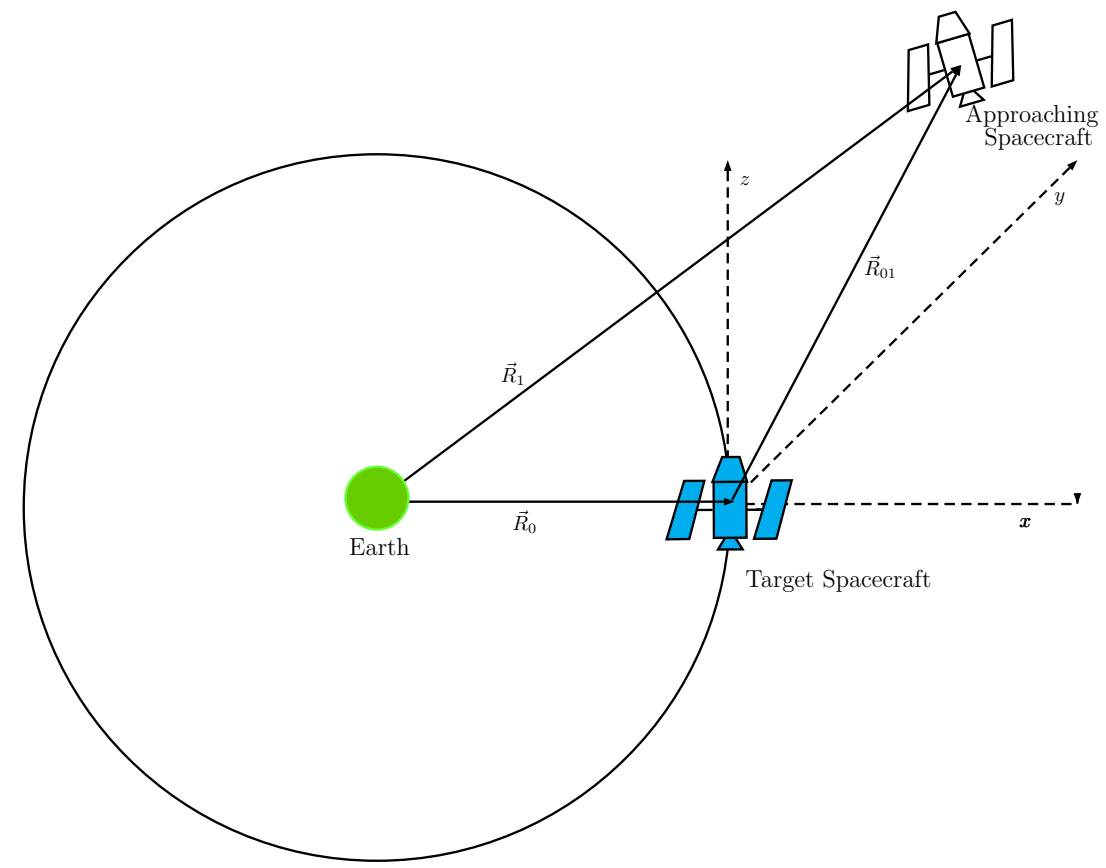

Figure 1. Hill's Frame

control index $J$ subject to constraints $\vec{C} \in \mathbb{R}^{m_{c}}$. To summarize an optimal control problem is solved at each time instant $t$ given by [8]:

$$
\begin{aligned}
\min _{\mathbf{u}} J & =\varphi\left(\vec{x}_{\tau}(T, t), \vec{p}(T, t)\right) \\
& +\int_{t}^{t+T} L\left(\vec{x}_{\tau}(\tau, t), \vec{u}_{\tau}(\tau, t), \vec{p}(\tau, t)\right) d \tau \\
\text { s.t. } \quad \dot{\vec{x}}_{\tau}(\tau, t)= & \vec{f}\left(\vec{x}_{\tau}(\tau, t), \vec{u}_{\tau}(\tau, t), \vec{p}(\tau, t)\right) \\
& \vec{x}_{\tau}(0, t)=\vec{x}(t) \\
& \overrightarrow{0} \\
& \geq \vec{C}\left(\vec{x}_{\tau}(\tau, t), \vec{u}_{\tau}(\tau, t), \vec{p}(\tau, t)\right) .
\end{aligned}
$$

where $\varphi$ is the terminal cost, $L$ is the current cost and $\vec{x}_{\tau}$, $\vec{u}_{\tau}$ are the predicted trajectories indexed by $\tau$ in the prediction horizon. In the above optimal control problem the feedback in the closed loop system is obtained by the equation (7), where the actual state $x(t)$ of the system is used as initial state $\vec{x}_{\tau}(0, t)$.

In the current paper the continuation generalized minimum residual method (CGMRES)[8] is used to solve the optimal control problem. The CGMRES method has been implemented and discussed in [9], [10], [11], [12] and [13].

\section{Simulation}

The application of Model Predictive Control (MPC) to the problem of Spacecraft Rendezvous is discussed here. The Clohessy-Wiltshire ( $\mathrm{CW}$ ) equation which was discussed earlier is used for simulation with the constant $n_{0}=0.0011$. Two simulation scenarios are considered: First the MPC technique is used for trajectory control with fuel efficiency and secondly the trajectory control problem is handled including collision avoidance in orbit.

\subsection{Trajectory Control with Fuel Efficiency}

The first scenario to be studied is the trajectory control problem. Here the spacecraft starts from initial condition $\left[x_{i}, y_{i}, z_{i}\right]=[100,100,100]$ to a final rendezvous point $\left[x_{r}, y_{r}, z_{r}\right]=[5,5,5]$ metres.

The cost function used in the current scenario is as follows:

$$
J_{1}=e_{p}^{T} Q e_{p}+u^{T} R u-g \mathbf{v}
$$

where $e_{p}$ is the position error, $-50 \leq u \leq 50$ is the control input and $\mathbf{v}$ is the vector of slack variables. Here $Q=$ $\operatorname{diag}[10,10,10,10,10,10], R=\operatorname{diag}[10,10,10,0,0,0]$ are respectively position and control performance gains and $g$ is the Lagrange multiplier gain.

The simulation results for the current trajectory control scenario can be seen in Figure-2. We can respectively see the position $(x, y, z)$, velocity $(\dot{x}, \dot{y}, \dot{z})$ and control input $\left(u_{x}, u_{y}, u_{z}\right)$ in Figure-2. In Figure-3 we can see the fuel consumption for different penalty. We can observe that a higher fuel efficiency can be obtained by larger penalty.

\subsection{Trajectory Control with Collision Avoidance}

The second scenario is the case where trajectory control problem is performed with collision avoidance. Here the spacecraft starts from initial condition $\left[x_{i}, y_{i}, z_{i}\right]=$ $[100,100,100]$ and has to reach a final rendezvous point $\left[x_{r}, y_{r}, z_{r}\right]=[5,5,5]$ metres with an obstacle at position $[50,50,50]$. It is assumed that the exact position of the obstacle is available as measurement to the controller on the spacecraft.

The cost function used in the current trajectory control with collision avoidance problem is as follows :

$$
J_{1}=e_{p}^{T} Q e_{p}+u^{T} R u-g \mathbf{v}+e^{-k \gamma}
$$



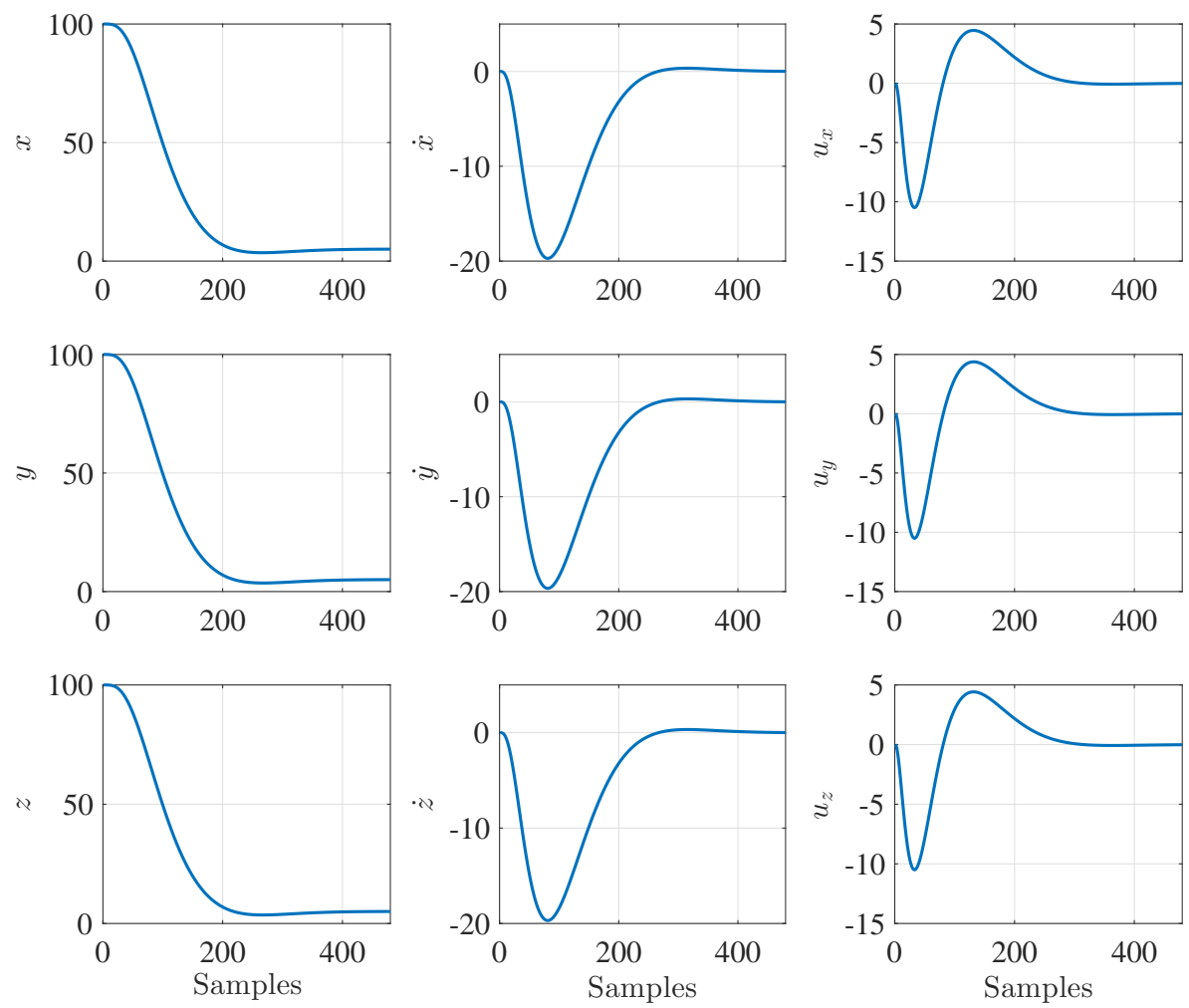

Figure 2. Rendezvous control with high control penalty $R=\operatorname{diag}[10,10,10,0,0,0]$.

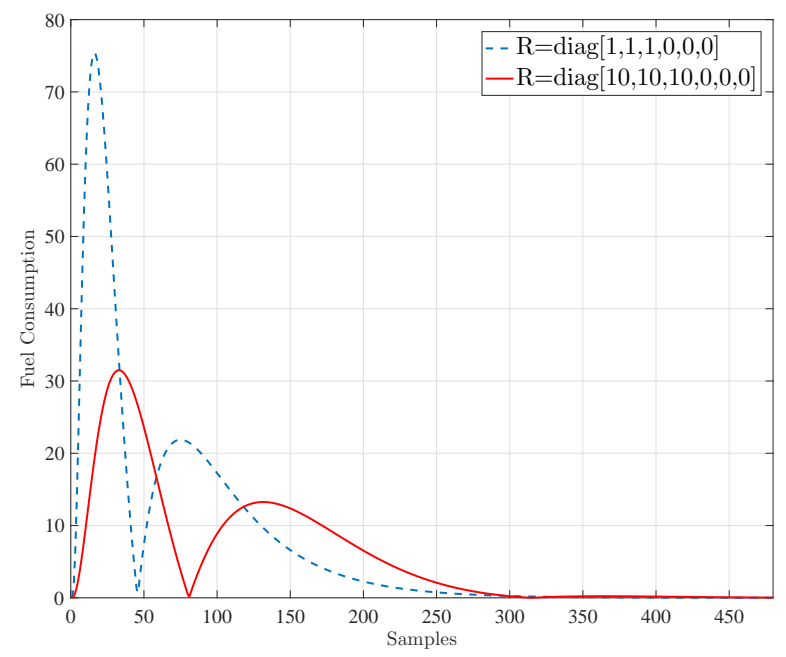

Figure 3. Rendezvous control: fuel efficiency

where $e_{p}$ is the position error, $-50 \leq u \leq 50$ is the control input and $\mathbf{v}$ is the vector of slack variables and $\gamma$ is given by

$$
\gamma=\left(x-x_{o}\right)^{2}-x_{c}^{2}+\left(y-y_{o}\right)^{2}-y_{c}^{2}+\left(z-z_{o}\right)^{2}-z_{c}^{2}
$$

where $(x, y, z)$ is the position of the spacecraft, $\left(x_{o}, y_{o}, z_{o}\right)$ is the position of the obstacle, $\left(x_{c}, y_{c}, z_{c}\right)$ is the clearance value. Here $Q, R$ are respectively position and control performance gains and $g$ is the Lagrange multiplier gain and $k$ is a tuning gain used in the collision avoidance term $e^{-k \gamma}$.

The simulation results for the current trajectory control with collision avoidance scenario can be seen in Figure-5

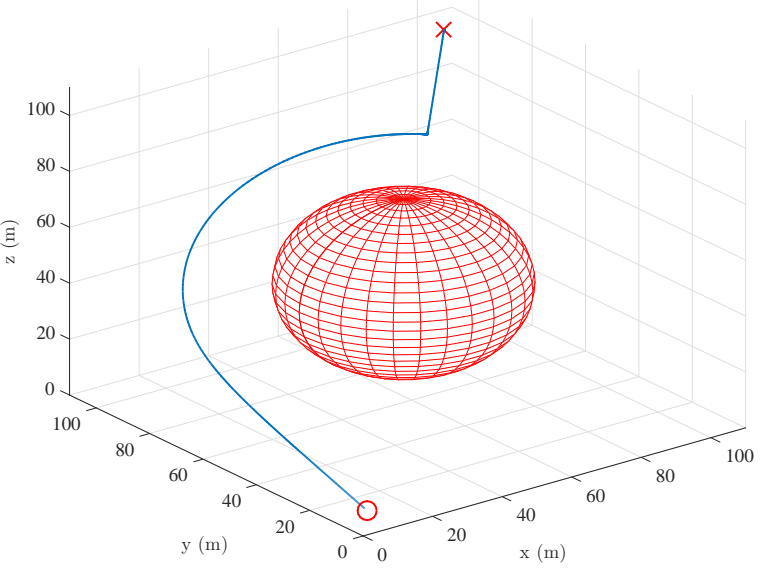

Figure 4. Rendezvous control: Collision Avoidance Trajectory, $\circ$ is the final position and $x$ is the initial point.

and Figure-4. We can respectively see the position $(x, y, z)$, velocity $(\dot{x}, \dot{y}, \dot{z})$ and control input $\left(u_{x}, u_{y}, u_{z}\right)$ in Figure-5. Similarly in Figure- 4 we can see the trajectory plot with the obstacle.

\section{Conclusion}

The problem of spacecraft rendezvous was discussed using simulation studies on the Clohessy-Wiltshire equations. The Model Predictive Control technique was used to perform trajectory control with collision avoidance using soft 

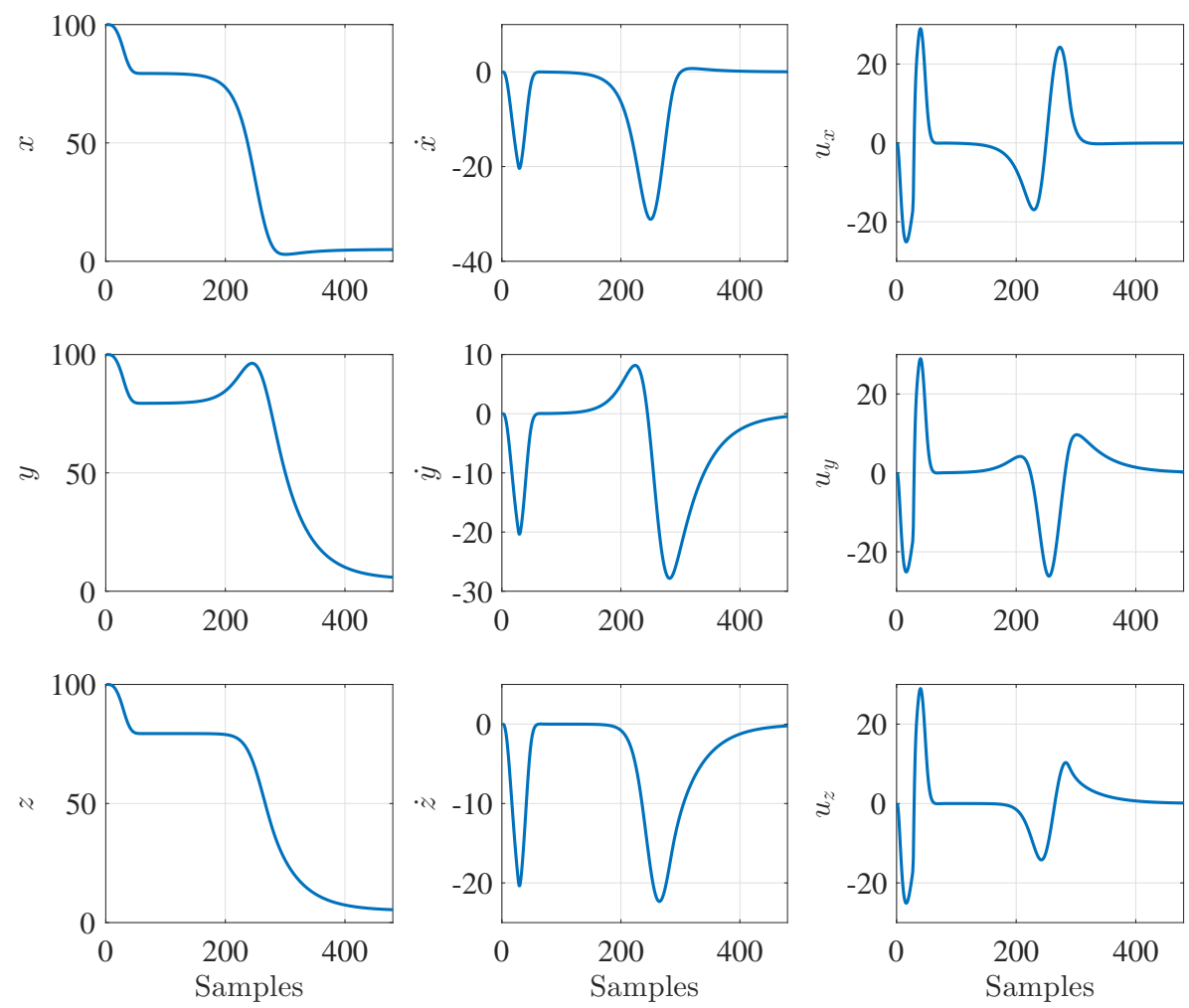

Figure 5. Rendezvous control: Collision Avoidance

constraint method. The simulation studies successfully validated the proposed control methodology.

\section{References}

[1] Y. Luo, J. Zhang, G. Tang, Chinese Journal of Aeronautics 27, 1 (2014)

[2] E.N. Hartley, A tutorial on model predictive control for spacecraft rendezvous, in Control Conference (ECC), 2015 European (2015), pp. 1355-1361

[3] C. Jewison, R.S. Erwin, A. Saenz-Otero, Model Predictive Control with Ellipsoid Obstacle Constraints for Spacecraft Rendezvous, in 1st IFAC Workshop on Advanced Control and Navigation for Autonomous Aerospace Vehicles ACNAAV'15 (2015)

[4] A. Weiss, M. Baldwin, R.S. Erwin, I. Kolmanovsky, IEEE Transactions on Control Systems Technology 23, 1638 (2015)

[5] R. Vazquez, F. Gavilan, E.F. Camacho, IFACPapersOnLine 48, 251 (2015)

[6] M.J. Sidi, Spacecraft Dynamics and Control (Cambridge University Press, 1997)

[7] R. Dai, J. Maximoff, M. Mesbahi, IEEE Transactions on Aerospace and Electronic Systems 49, 1968 (2013)
[8] T. Ohtsuka, Automatica 40, 563 (2004)

[9] S.A. Sajadi-Alamdari, H. Voos, M. Darouach, Nonlinear model predictive extended eco-cruise control for battery electric vehicles, in 2016 24th Mediterranean Conference on Control and Automation (MED) (2016), pp. 467-472

[10] J. Dentler, S. Kannan, M.A.O. Mendez, H. Voos, A real-time model predictive position control with collision avoidance for commercial low-cost quadrotors, in 2016 IEEE Multi-Conference on Systems and Control (MSC 2016) (2016)

[11] H. Seguchi, T. Ohtsuka, Nonlinear receding horizon control of an RC hovercraft, in Control Applications, 2002. Proceedings of the 2002 International Conference on (2002), Vol. 2, pp. 1076-1081 vol.2

[12] H. Seguchi, T. Ohtsuka, International Journal of Robust and Nonlinear Control 13, 381 (2003)

[13] J. Dentler, S. Kannan, M.A.O. Mendez, H. Voos, $A$ modularization approach for nonlinear model predictive control of distributed fast systems, in 2016 24th Mediterranean Conference on Control and Automation (MED) (2016), pp. 292-297 